\title{
Hospital-acquired pneumonia in an intensive care unit in Vietnam: clinical characteristics and pathogenic bacteria
}

Thao T.B. Nguyen ${ }^{1}$,

Kien T. Nguyen ${ }^{2}$,

Suol T. Pham ${ }^{3 *}$,

Xuan D. Pham ${ }^{4}$,

Thang Nguyen ${ }^{3}$

${ }^{1}$ Department of Intensive Care, Can Tho General Hospital, Can Tho, Vietnam

${ }^{2}$ Department of Physiology, Can Tho University of Medicine and Pharmacy, Can Tho, Vietnam

${ }^{3}$ Department of Pharmacology and Clinical Pharmacy, Can Tho University of Medicine and Pharmacy, Can Tho, Vietnam

${ }^{4}$ Faculty of Pharmacy, Can Tho University of Medicine and Pharmacy, Can Tho, Vietnam

*Corresponding author:

Suol T. Pham

ptsuol@ctump.edu.vn

\section{KEYWORDS:}

Hospital-acquired pneumonia

(HAP); Ventilator-associated pneumonia (VAP); Intensive Care Unit (ICU); Clinical characteristics; Pathogenic bacteria

https://www.pharmacy.mahidol.ac.th/journal/ (C) Faculty of Pharmacy, Mahidol University (Thailand) 2020

\begin{abstract}
The increase in the number of multi-drug resistant bacteria causing nosocomial infections at the hospital's intensive care unit (ICU) is a major worldwide problem. This leads to the need for research on clinical characteristics and pathogenic bacteria of hospital-acquired pneumonia (HAP) patients. Our research aimed to describe the clinical characteristics and identify the patterns of causative agents in HAP patients. We conducted a cross-sectional descriptive study on 103 HAP patients treated in ICU of a major hospital in Can Tho City, Vietnam from May 2017 to May 2018. The identification and antimicrobial susceptibility of cultures were determined using an automated microbiology analyzer; the Bauer-Kirby disk diffusion method was used. Of 103 patients met the inclusion criteria, 95 patients were confirmed as presenting with HAP caused by MDR bacteria. The overall prevalence was higher in men than in women and increased with age. There was some evidence of a correlation between prior broad-spectrum antibiotic use and the incidence of HAP. The sputum color change and hyperthermia were common clinical symptoms in HAP diagnosing. Notably, the proportion of multiple-antibiotic-resistant bacteria causing HAP was $92.5 \%$. High rates of most common antibiotic resistance were observed in Acinetobacter baumannii, Klebsiella, Pseudomonas aeruginosa and also E. coli. The success rate of treatment for HAP caused by MDR bacteria accounted for $49.5 \%$. The resistance rates of Acinetobacter baumannii and Pseudomonas aeruginosa to most common antibiotics were increasing substantially. The initial treatment for HAP ought to comply with combined antibiotic therapy or a new treatment strategy.
\end{abstract}

\section{INTRODUCTION}

Hospital-acquired pneumonia (HAP) and multi-drug resistant (MDR) bacteria in the intensive care unit (ICU) are developing countries' problems with high mortality rates (30 $70 \%{ }^{1,2}$. HAP is a lower respiratory infection that was not incubating at the time of admission and presents after more than 48 hours since hospitalization. HAP prolongs the duration of treatment, increases the costs of lab tests, and requires costly broad-spectrum antibiotics. According to WHO studies in high-income countries, 
the attributable costs at 10,000-25,000 US dollars per VAP case ${ }^{3}$. The costs of HAP is a massive financial burden for healthcare systems, especially Vietnam-a lower-middle-income country (LMICs) ${ }^{1,3}$.

A systematic review and meta-analysis of VAP in adults in Asia indicated that resistant gram-negative bacteria were the major cause of VAP in Asia. The most common organisms were Acinetobacter baumannii (26\%), Pseudomonas aeruginosa (22\%) and Klebsiella pneumonia (14\%), in which A. baumannii was the most common agent in the LMICs and tropical regions ${ }^{4}$. Other studies have demonstrated that Acinetobacter baumannii (24.4\%), Pseudomonas aeruginosa (13.8\%), and Klebsiella pneumonia (11.6\%) were the most common bacteria in ICU in Vietnam ${ }^{1}$. In Can Tho, 2016, the percentage of mentioned gram-negative bacteria were $44.7 \%, 15.4 \%$, and $9.8 \%$, respectively. Those figures changed to $48.9 \%, 19.8 \%$ and $6.5 \%$ in $2017^{5}$.

Recently, it is difficult to treat HAP due to the increase of antibiotic resistance and the lack of new antibiotic generations ${ }^{6}$. The number of reports about bacterial etiology and MDR bacteria is increasing. Now, ICUs in Asia are highly resistant to antibiotics by methicillin-resistant Staphylococcus aureus, MDR P. aeruginosa and A. baumannii ${ }^{1,4}$. In Asia, the resistance rates of $A$. baumanii, $P$. aeruginosa to carbapenem were $58 \%$ and $41 \%$ for LMICs, respectively. According to research in ICU in Vietnam, carbapenem resistance was predominant in A. baumannii (89.2\%) and Pseudomonas aeruginosa (55.7\%). Whilst, 14.9\% Klebsiella pneumonia were carbapenem-resistant and more than $75 \%$ of the Staphylococcus aureus isolates were methicillin-resistant ${ }^{1}$.

Studying about clinical characteristics and bacteria antibiotic resistance becomes urgent and necessary. However, causative agents and antibiotic resistance alter in accordance to ICU and period of time. Therefore, it is complicated to apply a general treatment guideline. From mentioned clinical difficulties, our research was conducted to determine clinical characteristics and pathogenic bacteria and other related factors and assess the treatment results on HAP patients in an ICU in Vietnam.

\section{MATERIALS AND METHODS}

\subsection{Study population}

The study had been conducted on all patients diagnosed with HAP and aged 32 to 94 years old in ICU of one of the top-tier hospitals of Can Tho City, Vietnam from May 2017 to May 2018. The hospital underwent a more-than-120-year-ofexistence history, providing leading health care services to Mekong Delta dwellers. The hospital admits about 1,700 patients a day and currently has 700 beds, but it is also constantly overloaded with patients. The bed occupancy rate often reaches at $113 \%$ and the number of visitors entering hospital peaks at $148 \%$ of the planned target. With firstclass facilities, cutting-edge equipment and a team of highly skilled doctors, the hospital is increasingly improving the quality of diagnosis and treatment for patients. The study was approved for implementation by the Research Ethics Committee of Can Tho University of Medicine and Pharmacy according to the minutes No. 20170417/CTUMP.

\subsection{Study design}

We carried out this cross-sectional descriptive study on a sample of $103 \mathrm{ICU}$ patients and had the informed consents from them and their relatives if the patients could not sign.

\subsection{Inclusion and exclusion criteria}

Inclusion criteria were patients who had 48 hours of hospital admission and presented a new or progressive radiographic infiltrate with at least two of four clinical features: (1) hyperthermia (i.e., fever) greater than $38^{\circ} \mathrm{C}$ or hypothermia less than $36^{\circ} \mathrm{C}$, (2) leukocytosis (circulating leukocyte count greater than $11 \mathrm{G} / \mathrm{L}$ ) or leukopenia (less than $4 \mathrm{G} / \mathrm{L}$ ), (3) increased sputum or changes in the properties, (4) decline in oxygenation (emergence of acute respiratory failure in patients with stable respiratory conditions). Patients, with bronchoalveolar lavage fluid over $1 \times 10^{4} \mathrm{cfu} / \mathrm{mL}$, bacteria isolated from blood cultures or pleural fluid, or present bacteria and antibiogram in bacteria culture results, were also included in the study.

Patients were excluded if they met one of the following criteria: diagnosed with communityacquired pneumonia, terminal cancer, AIDS, pregnant women or being treated with pulmonary tuberculosis $(+)$. 


\subsection{Sample size and sample selection}

The sample size is calculated by the formula: $n=\frac{Z_{(1-\alpha / 2)}^{2} p(1-p)}{c^{2}}$

Where: $n$ : the number of studied patients ( $\mathrm{n}=103$ after calculating)

$\alpha$ : probability of type 1 errors $(\alpha=0.05)$

$\mathrm{Z}$ : normal distribution value $(\mathrm{Z}$-score $=1.96$ for $\alpha=0.05)$

c: allowable error $=0.05$

$\mathrm{p}$ : The rate of multi-resistant bacteria causing HAP $(\mathrm{p}=92.8 \%$ according to research by Nguyen Ngoc Dai Trang ${ }^{7}$ )

We used the non-probability convenient sampling technique to select patients to participate in the study.

\subsection{Data collection}

The data including demographics, intrinsic and extrinsic risk factors for pneumonia, clinical signs and symptoms, isolated pathogens with antibiogram results, and outcome on discharge from the hospitals (i.e., the results of recovery, degeneration or death) were collected by structured questionnaires and medical records.

\subsection{Examination and sample collection procedure}

The initial examinations and laboratory tests, including routine biochemistry and specialized tests, were done when patients were first admitted to ICU. The daily follow-up examinations were performed to diagnose HAP on all ICU patients, including patients from other departments or route admitted within 48 hours. When the patients met the inclusion criteria, they would have clinical examinations to detect risk factors and severe prognostic signs. An empiric antibiotic therapy based on hospital's protocol was used when the sputum cultures and antibiogram results were available.

In the treatment of MDR pathogens, the empirical antibiotic therapy (Appendix 1), the duration of treatment and the dosage of antibiotics (Appendix 2) were based on Vietnam Association Against Tuberculosis and Lung Diseases (VATLD)'s guideline ${ }^{7}$. If the initial antibiotic agent was appropriate, or the pathogen was not Pseudomonas aeruginosa or Acinetobacter baumannii, or there was the patient's clinical symptom improvement, the duration of treatment was from 7 to 10 days. The duration would be longer (from 14 to 21 days) in case the MDR bacteria were Pseudomonas aeruginosa or Acinetobacter baumannii, with prolonged clinical symptoms such as fever (greater than $38^{\circ} \mathrm{C}$ ), circulating leukocyte count greater than $10,000 / \mathrm{mm}^{3}$, purulent sputum, and there was no improvement in chest X-ray results ${ }^{7}$.

We recorded the following clinical symptoms on patients: fever, and increased sputum or changes in the properties. All sputum specimens were collected by expectoration and stored in sterilized vials. Specimens were considered as standard if there were more than 25 polymorphonuclear leukocytes or less than 10 epidermal cells. The results were considered as positive when the number of observed bacteria was more than $10^{6}$ per $1 \mathrm{~mL}$ of sputums.

The bronchoalveolar lavage (BAL) fluid was collected from all intubated patients by bronchoscopy procedure and then transferred to the microbiology department within 10 minutes after collection. A sample was considered as positive and statistical significant if there were more than $10^{4}$ cfu per milliliter of BAL ${ }^{9}$. The routine identification of bacteria by the Department of Microbiology of the study hospital, identification of bacteria by Vitek 2 Compact machine, cultured by quantitative methods using a $10 \mu \mathrm{L}$ implant, results considered to be significantly positive with a threshold of $\geq 10^{4}$ bacteria $/ \mathrm{mL}$ of the sample. The disk-diffusion agar method was based on the Clinical and Laboratory Standard Institute guidelines (CLSI, 2014). The diameter of sterile ring around the antibiotic paper disks was measured and compared with the inhibition zone diameter standard. The bacteria was defined as MDR when the antibiogram result showed resistance to at least one agent in three or more different classes of antibiotics ${ }^{10}$ (Appendix 3). 


\subsection{Data analysis}

Descriptive statistics were used to conduct statistical analyses in our study. The qualitative variables were described by frequency, percentage, and $95 \%$ confidence interval; the quantitative variables were described by mean (or median), standard deviation (or quartile), minimum and maximum. The averages were compared by the T-test (if there was a normal distribution) or Wilcoxon (if there was no normal distribution); the difference between groups' ratios was compared by a chi-square test and corrected by Fisher'exact when the expected frequency is $<5$. The Odds Ratio (OR) was estimated to find the effect of variables on the outcome of multiple antibiotic resistance in bacteria.

\subsection{Statistical analysis}

All analyses were conducted using SPSS version 22.0. The difference between groups was considered as significant if the $p$-values were $<0.05$.

\section{RESULTS AND DISCUSSION}

3.1. Clinical characteristics and the association between baseline characteristics and multi-drug resistance

Of 103 patients met the inclusion criteria, 95 patients were confirmed as presenting with HAP caused by MDR bacteria. Fifty-seven percent of the patients were women. The overall prevalence was higher in men than in women and increased with age. For the oldest patients ( $>80$ years old), we found a prevalence of $42.7 \%$ versus $1.9 \%$ for the youngest patients ( $16-39$ years old) (Table 1$)$. Similar to the other study in Vietnam, old age is related to the occurrence of $\mathrm{HAP}^{11}$. The elderly usually suffers from chronic diseases, e.g., diabetes, hypertension, chronic obstructive pulmonary disease, which are risk factors causing immunodeficiency and secretion stagnation. However, there was no statistical difference between the incidence of MDR HAP and patient characteristics (i.e., gender, age, and types of pneumonia with $\mathrm{p}=0.07,0.651$, and 0.468 , respectively) (Table 1).

The risk factors for HAP are also shown in Table 1. According to the research from Seligman et al., prior broad-spectrum antibiotic usage within 10 days was an independent risk factor $(\mathrm{OR}=3.45$, $95 \%$ CI: $1.56-7.61, p=0.002)^{12}$. In our study, patients who used broad-spectrum antibiotics prior to the time of diagnosis had a higher incidence risk (98.1\%) than those who did not $(86.3 \%)$. This difference reached a statistical significance $(\mathrm{p}=0.031)$. However, there was no significant difference between MDR and non-MDR HAP in the viewpoint of factors as follows: previous antibiotic administration, re-hospitalization and blood filtration, immunodeficiency $(\mathrm{p}>0.05)$ (Table 1).

Table 1. The association between baseline characteristics and HAP caused by bacteria

\begin{tabular}{|c|c|c|c|c|}
\hline Baseline characteristics & $\begin{array}{c}\text { HAP caused } \\
\text { by MDR } \\
\text { bacteria } n(\%)\end{array}$ & $\begin{array}{c}\text { HAP caused by } \\
\text { non-MDR } \\
\text { bacteria n(\%) }\end{array}$ & $\begin{array}{c}\text { OR } \\
95 \% \text { CI }\end{array}$ & p-value* \\
\hline \multicolumn{5}{|l|}{ Gender } \\
\hline Male & $38(86.4)$ & $6(13.6)$ & 0.222 & \multirow{2}{*}{0.07} \\
\hline Female & $57(96.6)$ & $2(3.4)$ & $(0.04-1.16)$ & \\
\hline \multicolumn{5}{|l|}{ Age } \\
\hline $16-39$ & $2(100.0)$ & $0(0.0)$ & \multirow{4}{*}{0.651} & \\
\hline $40-59$ & $13(86.7)$ & $2(13.3)$ & & \\
\hline $60-79$ & $38(90.5)$ & $4(9.5)$ & & \\
\hline$\geq 80$ & $42(95.5)$ & $2(4.5)$ & & \\
\hline \multicolumn{5}{|l|}{ Types of pneumonia } \\
\hline HAP & $52(94.5)$ & $3(5.5)$ & 0.222 & \multirow{2}{*}{0.468} \\
\hline VAP & $43(89.6)$ & $5(10.4)$ & $(0.04-1.16)$ & \\
\hline
\end{tabular}


Table 1. The association between baseline characteristics and HAP caused by bacteria (cont.)

\begin{tabular}{|c|c|c|c|c|}
\hline Baseline characteristics & $\begin{array}{c}\text { HAP caused } \\
\text { by MDR } \\
\text { bacteria n(\%) }\end{array}$ & $\begin{array}{c}\text { HAP caused by } \\
\text { non-MDR } \\
\text { bacteria n(\%) }\end{array}$ & $\begin{array}{c}\text { OR } \\
95 \% \mathrm{CI}\end{array}$ & p-value* \\
\hline \multicolumn{5}{|l|}{ Time of onset of HAP } \\
\hline Late & $59(95.2)$ & $3(4.8)$ & 2.73 & \multirow{2}{*}{0.26} \\
\hline Early & $36(87.8)$ & $5(12.2)$ & $(0.6-12.1)$ & \\
\hline \multirow{2}{*}{\multicolumn{5}{|c|}{$\begin{array}{l}\text { Antimicrobial therapy } \\
\text { in the preceding } 90 \text { days }\end{array}$}} \\
\hline & & & & \\
\hline Yes & $20(95.2)$ & $1(4.8)$ & 1,87 & \multirow{2}{*}{1.00} \\
\hline No & $75(91.5)$ & $7(8.5)$ & $(0.3-16.1)$ & \\
\hline \multicolumn{5}{|c|}{ Residence in a nursing home } \\
\hline Yes & $6(100.0)$ & $0(0.0)$ & & \multirow{2}{*}{1.00} \\
\hline No & $89(91.8)$ & $8(8.2)$ & - & \\
\hline \multicolumn{5}{|c|}{ Re-hospitalization in the preceding } \\
\hline \multicolumn{5}{|l|}{90 days } \\
\hline Yes & $31(96.9)$ & $1(3.1)$ & 3.4 & \multirow{2}{*}{0.43} \\
\hline No & $64(90.1)$ & $7(9.9)$ & $(0.4-28.8)$ & \\
\hline \multicolumn{5}{|c|}{ Blood filtration in the preceding } \\
\hline \multicolumn{5}{|l|}{90 days } \\
\hline Yes & $7(100.0)$ & $0(0.0)$ & \multirow[b]{2}{*}{-} & \multirow{2}{*}{1.00} \\
\hline No & $88(91.7)$ & $8(8.3)$ & & \\
\hline \multicolumn{5}{|l|}{ Immunodeficiency } \\
\hline Yes & $6(100.0)$ & $0(0.0)$ & \multirow{2}{*}{-} & \multirow{2}{*}{1.00} \\
\hline No & $88(91.8)$ & $8(8.2)$ & & \\
\hline \multirow{2}{*}{\multicolumn{5}{|c|}{$\begin{array}{l}\text { Broad-spectrum antibiotics } \\
\text { used before }\end{array}$}} \\
\hline & & & & \\
\hline Yes & $51(98.1)$ & $1(1.9)$ & 8.1 & \multirow{2}{*}{0.031} \\
\hline No & $44(86.3)$ & $7(13.7)$ & $(0.96-68.5)$ & \\
\hline
\end{tabular}

Abbreviations: HAP, hospital-acquired pneumonia; MDR, multi-drug resistant; OR, odds ratio; CI, confidence interval.

The increase and color change of sputum usually indicates the causes of HAP, which are bacteria. The increase was observed in 82 cases $(86.3 \%)$. The most common color is off-white $(41.1 \%)$, followed by yellow (32.6\%) and green $(12.6 \%)$ (Table 2). Our result is comparable to that of the research in Vietnam (with the proportion of the first two kinds is $47.7 \%$ and $36.4 \%$, respectively $)^{11}$. This change is a crucial clinical symptom in HAP diagnosing ${ }^{6}$.
Fever is another clinical symptom, but in our research, this symptom appeared in $63 \%$ of patients, with more than $58 \%$ of cases have body temperature above $38.5{ }^{\circ} \mathrm{C}$ (Table 2). The figure for hyperthermia was slightly lower than other studies. This may be related to the characteristics (i.e., old age) of our study population. The elderly with a degradative health condition and using prior combined antibiotic therapy might not present fever symptoms, sometimes experienced hypothermia. 
Table 2. The features of fever and sputum symptom in HAP patients

\begin{tabular}{lccl}
\hline \multicolumn{1}{c}{ Features } & $\begin{array}{c}\text { Number of } \\
\text { patients (n=95) }\end{array}$ & Percentage (\%) & Notes \\
\hline Temperature & & & Mean \pm SD: $38.2^{\circ} \mathrm{C} \pm 1.06 \mathrm{SD}$ \\
$\quad$ Hyperthermia $(\geq 38 \mathrm{oC})$ & 60 & 63.2 & Lowest temperature: $36.5^{\circ} \mathrm{C}$ \\
$\quad$ No fever & 35 & 36.8 & Highest temperature: $40^{\circ} \mathrm{C}$ \\
Increase of sputum & & & \\
No & 13 & 13.7 & \\
Yes & 82 & 86.3 & \\
$\quad$ Off-white & 39 & 41.1 & \\
$\quad$ Yellow & 31 & 32.6 & \\
$\quad$ Green & 12 & 12.6 & \\
\hline
\end{tabular}

Abbreviations: SD, standard deviation.

\subsection{The proportion of antibiotic-resistant bacteria}

Of 107 isolated bacteria causing HAP, the percentage of MDR bacteria was $92.5 \%$ and the rest was non-MDR bacteria. Gram-negative bacteria accounted for the largest proportion of MDR bacteria (95\%; Table 3). This high rate of gram-negative bacteria was similar to that of other
Vietnam's researches ${ }^{13-15}$.

Out of the 99 MDR bacteria, Acinetobacter baumannii accounted for the highest proportion of $47.5 \%$. The percentage of Klebsiella pneumonia, Pseudomonas aeruginosa, and E. coli was $16.2 \%$, $12.1 \%$, and $10.1 \%$, respectively. Staphylococcus aureus is the only gram-positive bacteria that accounted for a low proportion (5.05\%) (Table 4).

Table 3. The percentage of isolated multiple-antibiotic-resistant bacteria

\begin{tabular}{ccc}
\hline Variable & Frequency $(\mathrm{n})$ & Percentage $(\%)$ \\
\hline MDR bacteria & 99 & 92.5 \\
Gram-negative bacteria & 94 & 95 \\
Gram-positive bacteria & 5 & 5 \\
Non-MDR bacteria & 8 & 7.5 \\
Total & 107 & 100 \\
\hline
\end{tabular}

Abbreviations: MDR, multi-drug resistant.

Table 4. The percentage of multiple antibiotic resistance based on classes of bacteria

\begin{tabular}{lccccc}
\hline \multicolumn{1}{c}{ Strains of isolated bacteria } & \multicolumn{2}{c}{ MDR } & \multicolumn{2}{c}{ Non-MDR } \\
& n & $\mathbf{\%}$ & $\mathbf{n}$ & $\mathbf{\%}$ \\
\hline Staphylococus aureus & 5 & 100.0 & 0 & 0.0 \\
$\quad$ Total of gram-positive bacteria & 5 & 100.0 & 0 & 0.0 \\
Acinetobacter baumannii & 47 & 100.0 & 0 & 0.0 \\
E. coli & 10 & 90.9 & 1 & 9.1 \\
Klebsiella pneumonia & 16 & 72.7 & 6 & 27.3 \\
Proteus spp & 2 & 100.0 & 0 & 0.0 \\
Pseudomonas aeruginosa & 12 & 92.3 & 1 & 7.7 \\
\hline
\end{tabular}


Table 4. The percentage of multiple antibiotic resistance based on classes of bacteria (cont.)

\begin{tabular}{lccccc}
\hline \multirow{2}{*}{ Strains of isolated bacteria } & \multicolumn{2}{c}{ MDR } & \multicolumn{2}{c}{ Non-MDR } \\
\hline Sphingomonas paucimobilis & $\mathbf{n}$ & $\mathbf{\%}$ & $\mathbf{n}$ & $\mathbf{\%}$ \\
Stenotrophomonas maltophilia & 1 & 100.0 & 0 & 0.0 \\
Enterobacter spp & 2 & 100.0 & 0 & 0.0 \\
Citrobacter freundii & 3 & 100.0 & 0 & 0.0 \\
$\quad$ Total of gram-negative bacteria & 1 & 100.0 & 0 & 0.0 \\
\hline
\end{tabular}

Abbreviations: MDR, multi-drug resistant.

\subsubsection{The antibiotic resistance of Acinetobacter} baumannii

The current study found that all of isolated Acinetobacter baumannii strains resisted to carbapenems, third-and-fourth-generation cephalosporins, and second-and-third-generation fluoroquinolones. In addition, all samples were resistant to several common antibiotics: combination of penicillin/beta-lactamase inhibitors (over 95\%), aminoglycosides (78-85\%), and co-trimoxazole (63.8\%) (Table 5). Similarly, the resistance rate to carbapenem, beta-lactam, cephalosporin was highly increasing according to other research in Vietnam ${ }^{11}$. This may be related to the widespread use of broad-spectrum antibiotics in ICU patients.

The susceptibility rates to colistin in Can Tho and Ho Chi Minh City were more than 90\% and $80 \%$, respectively ${ }^{5,16}$. In our study, colistin was the only drug that showed $100 \%$ sensitivity against all the species of $A$. baumannii, similar to research in ICU of Bach Mai Hospital that there was no emergence of colistin-resistant species reported ${ }^{17}$.

Table 5. The proportion of bacteria resistant to common antibiotics

\begin{tabular}{lccccc}
\hline Bacteria & $\begin{array}{c}\text { Acinetobacter } \\
\text { baumannii }\end{array}$ & $\begin{array}{c}\text { Klebsiella } \\
\text { pneumonia }\end{array}$ & $\begin{array}{c}\text { Pseudomonas } \\
\text { aeruginosa }\end{array}$ & E. coli & $\begin{array}{c}\text { Staphylococus } \\
\text { aureus }\end{array}$ \\
\hline Amikacin & 78.7 & 75 & 66.7 & 30 & 80 \\
Gentamicin & 83 & 75 & 91.7 & 40 & 60 \\
Tobramycin & 85.1 & 87.5 & 83.3 & 50 & 60 \\
Ampi/Sul & 100 & 75 & 75 & 70 & - \\
Cefepime & 100 & 87.5 & 83.3 & 80 & 80 \\
Cefotaxime & 100 & - & 100 & - & - \\
Ceftriaxone & 100 & 81.2 & 91.7 & 90 & - \\
Ceftazidime & 100 & 87.5 & 83.3 & 90 & - \\
Piper/Tazo & 97.9 & 75 & 75 & 80 & - \\
Ticar/Clavu & 95.8 & 81.2 & 91.7 & 100 & - \\
Imi/Cilastatin & 100 & 43.8 & 50 & 40 & 100 \\
Meropenem & 100 & 43.8 & 50 & 30 & 100 \\
Co-trimoxazole & 63.8 & 87.5 & - & 70 & - \\
Colistin & 0 & 18.2 & 16.7 & 0 & - \\
Ciprofloxacin & 100 & 62.8 & 83.3 & 70 & 100 \\
Levofloxacin & 100 & 62.5 & 75 & 90 & 80 \\
Ampicillin & - & 100 & - & - & - \\
Tigecycline & - & 0 & - & 0 & 0 \\
\hline
\end{tabular}


Table 5. The proportion of bacteria resistant to common antibiotics (cont.)

\begin{tabular}{lccccc}
\hline Bacteria & $\begin{array}{c}\text { Acinetobacter } \\
\text { baumannii }\end{array}$ & $\begin{array}{c}\text { Klebsiella } \\
\text { pneumonia }\end{array}$ & $\begin{array}{c}\text { Pseudomonas } \\
\text { aeruginosa }\end{array}$ & E. coli & $\begin{array}{c}\text { Staphylococus } \\
\text { aureus }\end{array}$ \\
\hline Ertibiotics* & - & - & 16.7 & 40 & - \\
Amox/Clavu & - & - & - & - & 80 \\
Clindamycin & - & - & - & - & - \\
Oxacillin & - & - & - & - & 80 \\
Vancomycin & - & - & - & - & 20 \\
Neltimicin & - & - & - & - & 0 \\
Linezolid & - & - & - & - & 0 \\
Teicoplanin & - & - & - & - & 0 \\
\hline
\end{tabular}

Abbreviations: Ampi/Sul, ampicillin and sulbactam; Piper/Tazo, piperacillin and tazobactam; Ticar/Clavu, ticarcillin and clavulanate; Imi/Cilastatin, imipenem and cilastatin; Amox/Clavu, amoxicillin and clavulanate.

*The isolated bacteria were assessed for their susceptibility to all suitable antibiotics available at the study hospital. All antibiotic agents appropriate for each type of bacteria isolated are shown in Table 5.

\subsubsection{The antibiotic resistance of Klebsiella}

The study demonstrated that Klebsiella was virtually resistant to broad-spectrum antibiotics: ampicillin (100\%), third-and-fourth-generation cephalosporins (over 80\%), a combination of penicillin/beta-lactamase inhibitors, e.g., piperacillin/ tazobactam (75\%), ticarcillin/clavulanic $(81,2 \%)$ (Table 5). Our research revealed that the cephalosporin and carbapenem resistance rate was higher than when compared to the research conducted in Asia and Europe ${ }^{4,18}$. These geographic differences in the rates of resistance may be in connection with national income, healthcare management systems and different patterns of antibiotic use.

Extended-spectrum $\beta$-lactamase (ESBL)producing Klebsiella was reported that having a cross-resistance between aminoglycoside and fluoroquinolone. This becomes a severe worldwide concern. Thus, carbapenem was a suitable choice for severe sepsis caused by ESBL-producing bacteria. However, K. pneumonia was highly resistant to carbapenem (nearly 50\%) when compared to it across Vietnam ${ }^{4}$. We couldn't explain a paradox that non-ESBL-producing Klebsiella rate was $68.7 \%$, it had low sensitivity to imipenem and meropenem (lower than 50\%). To answer, we need the genetic analysis of pathogenic bacteria in the future.

\subsubsection{The antibiotic resistance of Pseudomonas aeruginosa}

Pseudomonas aeruginosa ranked $3^{\text {rd }}(92.3 \%)$ in the microbiological isolation results of our study (Table 5). A high prevalence of Pseudomonas aeruginosa resistant to third-and-fourth-generation cephalosporins, second-and-third-generation fluoroquinolones (over 83\%), and carbapenems $(50 \%)$ was reported. Similar to a research in LMICs generally and in Vietnam particularly, the carbapenem resistance rate was $41 \%$ and $55.7 \%$, respectively ${ }^{1,4}$. Previously, the imipenem resistance rate in India was only approximately $10 \%$ and was having upward trends in resistance rate in either imipenem and cefoperazone-sulbactam, tazobactam, amikacin ${ }^{19}$. The sharply increased in resistance rates were reported as a result of antibiotic abuse, inappropriate antibiotic use strategies, and genetic mutation. Notably, all of the Pseudomonas aeruginosa strains were resistant to cefotaxime (100\%). Only a small fraction was resistant to all tested antibiotics.

\subsubsection{The antibiotic resistance of E. coli}

Similar to Klebsiella, E. coli is one of the common ESBL-producing strains. A high percentage of resistance to common antibiotics was found in the $E$. coli strains: third-and-fourth-generation cephalosporins ( $80-90 \%$ ), and second-and-thirdgeneration fluoroquinolones (over 70\%). E. coli was also resistant to the combination of penicillin/ beta-lactamase inhibitors with the proportion of $80 \%$. The present study showed a higher resistance rate of $E$. coli in comparison with the result from the study in Vietnam ${ }^{14}$. In that study, E. coli was 
virtually resistant to cephalosporins, fluoroquinolones (over $71 \%$ ) and was susceptible to the combination of penicillin/beta-lactamase inhibitors (e.g., piperacillin/tazobactam, ticarcillin/clavulanic) with the rate of $53 \%$.

Notably, E. coli was susceptible to tigecycline and colistin (resistance rate was $0 \%$ ) (Table 5), as same as what they found in $\mathrm{Nepal}^{20}$. The cephalosporin and carbapenem resistance in our study was quite similar to that in Asia. ( $77 \%$ and $27 \%$, respectively) ${ }^{4}$. The present study showed a similar resistance rate of $E$. coli in comparison with the result from the study of India, except for cephalosporin and carbapenem resistance rate ${ }^{19}$. In that study, the resistance rate was nearly $30 \%$ for ceftazidime and more than $10 \%$ for imipenem.

\subsubsection{The antibiotic resistance of Staphylococcus} aureus

Although Staphylococcus aureus strains accounted for a low proportion of MDR-bacteria $(5 \%)$ (Table 4), the bacteria were virtually resistant to common antibiotics (e.g., amoxicillin/clavulanic, cefepime, oxacillin). This high rate $(80 \%)$ was similar to other studies in Vietnam, in India and Asian countries ${ }^{7,15,19,21}$. However, there was an downward trend in resistance during 3 years. Our result was also consistent with the spread of antibiotic resistance in Asian countries. The MDR rate was $83.7 \%$. We found that all of the Staphylococcus aureus strains were sensitive to teicoplanin, linezolid, and tigecycline (Table 5). The resistance rates to almost antibiotics were high, but totally sensitive to vancomycin and teicoplanin ${ }^{21}$.

\subsection{The treatment results' assessment}

The results were assessed at the end of antibiotic treatments and classified into three groups. Group I was improvement cases using the initial antibiotics, group II was the number of recovered patients with alternative antibiotics, and the rest was no recovered cases, including failure cases with initial or alternative antibiotics. The figures for group II and group III were higher, at $34.7 \%$ and $35.8 \%$, respectively. The success rate of treatment for HAP caused by MDR bacteria accounted for only $49.5 \%$ (Table 6 ).

Table 6. Clinical improvement status with alternative antibiotics and the treatment results of HAP patients

\begin{tabular}{lcc}
\hline \multicolumn{1}{c}{ Results } & Number of patients $(\mathbf{n}=\mathbf{9 5})$ & Percentage $(\%)$ \\
\hline Clinical status & & \\
$\quad$ Recovered (no alteration) & 28 & 29.5 \\
Recovered & 33 & 34.7 \\
$\quad$ No recovered & 34 & 35.8 \\
Treatment results & & \\
$\quad$ Success & 47 & 49.5 \\
Failure & 48 & 50.5 \\
\hline
\end{tabular}

HAP's assessment and treatment are complicated tasks. The treatment must be taken immediately after clinical diagnosing based on patient's conditions. From the results of the clinical and paraclinical assessments, the proportion of recovered patients without changing antibiotics was $29.5 \%$ (Table 6 ). By contrast, $34.7 \%$ of patients recovered after alteration and the rest had degenerative clinical symptoms due to baseline or respiratory diseases, e.g., septic shock. Identical with other research from Chung, Luna et al., and AlvarezLerma, our results indicated inappropriate initial antibiotic treatment leads to higher fatal rate ${ }^{22-24}$.

\subsection{Limitations and strengths}

Our research has several limitations as follows. Firstly, the sample size was modest because the study was conducted in a local hospital; in addition, some cases were not recorded due to hospital referral or giving up treatment. We suggest that future research should be based on larger sample size and hence may take more years to complete. Lastly, in the disk diffusion test, only 
a few millimeters of difference in zone diameter separating susceptible from resistant strains resulted in a potentially ambiguous reading. Therefore, the experiment was conducted repeatedly to minimize the error rate. We suggest another testing method, in particular, minimum inhibitory concentration (MIC), should be applied for a better result.

One of the strengths is that our method (i.e., descriptive cross-sectional study) was appropriate to comprehend the MDR bacteria and influential factors in HAP at local scale. This method was used to figure out the predominant characteristics in a population at a specific period and provide an overall correlation between the incidence of HAP and risk factors that might exist. Besides, our method could be conducted in a short period of time with minimal resources, which is suitable for testing a new research hypothesis and paving the way for further experimental study. Finally, investigating the antibiotic resistance and clinical characteristics of HAP patients simultaneously provided practical and constructive information for medical practitioners as an internal guideline.

\subsection{Clinical application and future research}

Antimicrobial therapy plays an essential role in HAP treatment. Hence, grasping the resistance rate of bacteria in a specific community is necessary to initiate community-specific antibiotic treatment strategies. In fact, early and appropriate empirical antibiotic therapy is associated with an improvement in treatment outcomes and reduction in mortality. As an internal guideline, our research will guide the clinicians in choosing appropriate antibiotic strategies for the emergency treatment of severe sepsis. Because there was no record of colistin-resistant bacteria, colistin usage or colistin-tigecycline combined therapy are potential treatments for MDR HAP in the future.

\section{CONCLUSIONS}

In conclusion, these data suggested that coughing up sputum and the color change were crucial clinical symptoms in HAP diagnosing. Notably, the results of this study indicated an increasing trend in common antibiotic resistance of the Acinetobacter baumannii and Pseudomonas aeruginosa species, which are the major causes of MDR HAP. Aminoglycosides, cephalosporins, fluoroquinolones, and the combination of penicillin/beta-lactamase inhibitors were drastically ineffective.

\section{ACKNOWLEDGEMENTS}

Our gratitude to colleagues and students who participated in data analysis, drafting and revising the paper in the present study.

\section{Conflict of interest}

There are no interest conflicts during the study of this research.

\section{Funding}

None to declare

\section{Ethical approval}

None to declare

\section{Article info:}

Received November 3, 2019

Received in revised form January 13, 2020

Accepted February 12, 2020

\section{REFERENCES}

1. Phu, Vu Dinh, Wertheim, Heiman FL, Larsson, Mattias, et al. Burden of hospital acquired infections and antimicrobial use in Vietnamese adult intensive care units. PLoS One 2016 Jan 29;11(1):e0147544.

2. Ministry of Health. Antibiotic Guidelines for Ventilator-associated pneumonia. Ha Noi: Medical Publishing House; 2015. p. 100-8.

3. World Health Organization. Report on the Burden of Endemic Health Care-Associated Infection Worldwide: a system review of the literature. World Health Organization; 2011. p. 21.

4. Bonell, Ana, Azarrafiy, Ryan, Huong, Vu Thi Lan, et at. A systematic review and metaanalysis of ventilator-associated pneumonia in adults in Asia: an analysis of national income level on incidence and etiology. Clin Infect Dis 2019 Jan 18;68(3):511-8.

5. Nguyen Buu Huy. Microbiological analysis and antibiotic use on patients with hospital- 
acquired pneumonia in Can Tho General Hospital's intensive care unit [MPharm thesis]. Ha Noi: Ha Noi University of Pharmacy; 2018.

6. American Thoracic Society. Guidelines for the management for adults with Hospitalacquired, Ventilator - associated, and Healthcareassociated Pneumonia. Am J Respir Crit Med 2005;171:388-416.

7. Vietnam Association Against Tuberculosis and Lung Diseases. Guidelines on the management of non-tuberculous lower respiratory tract infections. Ha Noi: Medical Publishing House; 2012.

8. Nguyen Ngoc Dai Trang. Investigated the antibiotic resistance of ventilator-associated pneumonia at Cho Ray Hospital [Master thesis]. Ho Chi Minh City: University of Medicine and Pharmacy at Ho Chi Minh City; 2013.

9. Meduri GU, Chastre J. The standardization of bronchoscopic techniques for ventilatorassociated pneumonia. Infection Control \& Hospital Epidemiology. 1992 Nov;13(11):640-9.

10. Magiorakos A.P., Srinivasan A., Carey R.B., et al. Multidrug-resistant, extensively drugresistant and pandrug-resistant bacteria: an international expert proposal for interim standard definitions for acquired resistance. Clinical microbiology and infection, 2012, 18.3: 268-281.

11. La Quy Huong. A research of clinical and subclinical characteristics and assessment of therapeutic treatment in hospital associated pneumonia patients at respiratory center of Bach Mai hospital [Master thesis]. Ha Noi: Ha Noi University of Pharmacy; 2012.

12. Seligman, Renato, Ramos-Lima, Luis Francisco, Oliveira, Vivian do Amaral, et al. Risk factors for infection with multidrug-resistant bacteria in non-ventilated patients with hospital-acquired pneumonia. Jornal Brasileiro de Pneumologia 2013;39:339-48.

13. Pham Luc. Observation in vitro of bacteria causing hospital-required pneumonia at resuscitation and emergency department of Pham Ngoc Thach Hospital in 2010 - 2011. Ho Chi Minh Medical Journal 2013;17 Suppl 1:97-104.

14. Le Bao Huy, Nguyen Duc Thang. Ventilator associated pneumonia: the characteristics of agents and antibiotic resistance on the elderly patients at ICU of Thong Nhat hospital. Ho Chi Minh Medical Journal 2012;16 Suppl 1: 78-6.

15. Nguyen The Anh, Do Quyet. Characteristics of bacteria causing hospital-associated pneumonia and antibiotic resistance at ICU and emergency department of Huu Nghi hospital. Journal of Practical Pedicine 2011;798(12):3-5.

16. Le Tien Dung. Hospital-acquired pneumonia: characteristics and in-vitro resistance of bacteria at Ho Chi Minh City University Medical Center. [Online]. 2017 Nov 15; Available from: URL: http://www.hoihohaptphcm.org/ index.php/chuyende/benh-phoi/388-viem-phoibenh-vien-dhac-dhiem-vi-khuan-va-de-khangkhang-sinh-in-vitro-tai-benh-vien-dhai-hoc-yduoc-tphem.

17. Pham Hong Nhung, Dao Xuan Co, Bui Thi Hao. Susceptibility to antibiotics of gramnegative bacilli isolated in ICU of Bach Mai hospital. Journal of Medical Research 2017; 109(4).

18. Bianco A, Capano MS, Mascaro V, Pileggi C, Pavia M. Prospective surveillance of healthcareassociated infections and patterns of antimicrobial resistance of pathogens in an Italian intensive care unit. Antimicrobial Resistance \& Infection Control 2018 Apr 3;7:48.

19. Abhijit Chaudhury, A. Shobha Rani, Usha Kalawat, Sachin Sumant, Anju Verma, and B. Venkataramana. Antibiotic resistance \& pathogen profile in ventilator-associated pneumonia in a tertiary care hospital in India. Indian J Med Res 2016 Sep;144(3):440-6.

20. Narayan Prasad Parajuli, Subhash Prasad Acharya, Shyam Kumar Mishra, Keshab Parajuli, Basista Prasad Rijal and Bharat Mani Pokhrel. High burden of antimicrobial resistance among gram negative bacteria causing healthcare associated infections in a critical care unit of Nepal. Antimicrobial Resistance \& Infection Control 2017;6:67.

21. Song J-H,HsuehP-R, Chung DR, KoKS, Kang C-I, Peck KR, et al. Spread of methicillinresistant Staphylococcus aureus between the community and the hospitals in Asian countries: an ANSORP study. J Antimicrob Chemother 2011 Feb 20;66(5): 1061-9.

22. Chung, Song, Kim, Thamlikitkul V, Huang SG, Wang H, et al. High prevalence of multidrug- 
resistant nonfermenters in hospital-acquired pneumonia in Asia. Am J Respir Crit care Med 2011 Dec 15;184(12):1409-17.

23. Luna CM, Vujacich P, Niederman MS, Vay C, Gherardi C, Matera J, et al. Impact of BAL data on the therapy and outcome of ventilator- associated pneumonia. Chest 2012;111: 676-85.

24. Alvarez-Lerma F. Modification of empiric antibiotic treatment in patients with pneumonia acquired in the intensive care unit. Intensive Care Med 2016;22:387-94. 